\title{
The Mathematics of Nepotism: A Review of Foundations of Social Evolution by Steven A. Frank
}

\author{
Jason Noble \\ Informatics Research Institute \\ School of Computing, University of Leeds \\ Leeds LS2 9JT, United Kingdom \\ jasonn@ comp. leeds.ac.uk
}

The theory of natural selection suggests that animals will do their best to maximize their number of offspring. So why do some young Florida scrub jays stay at the nest and help their parents to raise next year's brood, instead of striking out on their own? Why do most ants, bees and termites remain childless, working tirelessly for the good of their colony? Ever since the landmark work of Hamilton (1964), we have understood such social phenomena in terms of kin selection. This is the idea that an organism has two ways of perpetuating its genetic material: firstly, by direct reproduction, and secondly, by assisting relatives in their reproductive efforts, thereby promoting the survival of copies of at least some of its genes. Hamilton quantified kin selection by noting that assisting a relative would be selected for if $r B-C>0$, that is, if the benefit of being helped out $(B)$, scaled by the degree to which the two parties were related $(r)$, was greater than the cost of being nice $(C)$.

Steven A. Frank, in his recent book Foundations of Social Evolution (1998), is not out to capsize this orthodox Hamiltonian view. However, he argues that the appealing simplicity of Hamilton's formulation can be misleading, and that it does not always help us to solve the problems we're interested in. For example, specifying exactly what is meant by relatedness has caused much confusion in the literature-Hamilton himself later revised his original definition (Hamilton, 1970; Michod \& Hamilton, 1980). It is also difficult to be clear and consistent about the units with which $B$ and $C$ should be measured.

Frank's goal is to clear up exactly these ambiguities. His book is about the proper measures of success or fitness needed to study social evolution. It is intended as both a practical guide to constructing mathematical models, and as a summary of the kin selection literature. The book also features original models and arguments. Frank focuses on three evolutionary currencies: marginal value, reproductive value, and inclusive fitness. The first two of these have familiar economic interpretations.

Marginal value is the rate of change in overall success as a given trait changes. Suppose that the trait under investigation is parental care, and too little of it leads 
to the death of unfortunate children, whereas too much wastes resources that could be used for future reproduction. A marginal value analysis simply notes that populations will experience selective pressure towards the intermediate optimum value of the trait. At equilibrium, success $(W)$ is maximized, and thus the equilibrium value of parental care $(t)$ is to be found by solving $\frac{d W}{d t}=0$. Of course, for some traits the optimum value will be zero or infinity (Williams, 1966). In these cases we expect selection for closer and closer approximations to the optimum, and a marginal value analysis will be inappropriate. Nevertheless, many traits involve the sort of tradeoff described above, where too much or too little is less than optimal.

Reproductive value is all about looking more closely at what "success" means in an evolutionary context. For example, having lots of children is a good thing, but not if they all die before reaching reproductive age. The quantity to be maximized is not, strictly speaking, number of offspring but genetic contribution to future generations, and the relevant variables include overall fecundity, early or late reproduction, parental investment, proportion of male and female offspring, etc. Frank quotes Fisher's (1930) characterization of reproduction as being similar to lending money: a newborn individual is loaned a life, and the question for evolutionary theorists is to determine the rate of interest payment (i.e., the rate of production of grandchildren) that would justify the loan's having been made in the first place.

Inclusive fitness is, approximately, the Hamiltonian idea described above, that genetic proliferation can be achieved both through direct reproduction and by assisting relatives. Inclusive fitness at first appears to be a concept unique to biological evolution. However, Frank argues both that the concept has not always been properly thought through in previous modelling efforts, and that it is closely linked to ideas on correlated equilibria in game theory. He claims that relatedness, the central idea in theories of inclusive fitness, is actually two things: a measure of correlation between the behaviours (or other phenotypic features) of social partners, and a measure of the fidelity of transmission of such traits to offspring. We will return to this important idea below.

The thesis is that through careful and simultaneous consideration of these three currencies we can construct mathematical models that help us to understand how cooperative social behaviour evolved. But, true to his name, Frank admits early on that the book is really about social natural selection rather than social evolution. By this he means that we cannot use the mathematical methods presented to make predictions about the grand sweep of evolutionary progress. Instead, given a particular context and various possible strategies, we can predict which genotypes will increase in frequency. The first step in doing so is to note that fitness depends not only on how well an individual does relative to those around it, but also on how well an individual's neighbourhood does relative to others. The key to understanding social evolution is in coming to terms with this tension between selfishness and cooperation.

So why should readers of Adaptive Behavior be interested in Foundations of Social Evolution? Many of those who read and contribute to this journal are concerned 
with problems that could be considered social, such as the origin of communication, or the best way to coordinate the behaviour of a group of robots. But our methods differ greatly from those of a theoretical biologist like Frank: adaptive behavior researchers typically use simulation or robotic models, in which a global outcome emerges from the interaction of simple local elements. In theoretical biology, on the other hand, a mathematical abstraction is used to model something global, such as the population mean value for a trait; it is taken on faith that low-level peculiarities will not invalidate the overall conclusion. This is not the place for a detailed comparison of the two research programs, but it seems safe to assume that they are complementary. Although simulation can capture fine-grained details that render mathematical treatments intractable, a simple mathematical model can sometimes be more elegant, more accessible, and, most importantly, more general than a complex simulation could ever be. In short, I would encourage Adaptive Behavior readers to look at Frank's book because it is important to know the competition. There is certainly room for both kinds of work, but ignorance on the part of an enthusiastic computer modeller can (and sometimes does) lead to the production of superfluous simulations when a mathematical model would be perfectly tractable and could have made the same point in a more general way.

\section{Mathematics made easy}

Another good reason for reading Frank's book is that he works hard to demystify mathematical notation. For anyone who has ever struggled through a theoretical biology paper with appendices resembling hieroglyphic inscriptions, Frank is profoundly reasssuring. Part of the problem, he says, is that terms like "additivity" and "relatedness" are used in different ways, notations are inconsistent, and there is a tendency for some authors to insist dogmatically that their variant is the right way to do things. Frank himself has a refreshingly pragmatic approach: through a discussion of the history of work on kin selection, focusing on models by Hamilton (1964) and Queller (1992), he makes it clear that a number of different ways of expressing the mathematics of evolving systems amount to the same thing. For example, the per-generation change in an evolving trait is often partitioned-we might say that this much of the change was due to selective effects, while that much was due to the process of transmission from parents to offspring. Other partitions include heritable vs. environmental components, or within- and between-group selective effects. Frank points out that these partitions are mathematically equivalent. It follows that there is no one right way to do things: the best partition is a matter of one's preferences and goals.

The book is certainly not light entertainment. The mathematics requires careful attention and some knowledge of calculus and matrix algebra, but it is very methodically presented and usually well explained. Perhaps one could make a case for the inclusion of a glossary, in which terms like "heritability" and "eigenvalue" were defined for the beginner, but clearly Frank had to assume some level of prior knowledge and could not cater to everyone. (With that in mind, if you have no idea what the terms "genetic covariance" or "additive model" mean, then it would probably be best to read an introductory text such as Maynard Smith (1989) before tackling Frank's more advanced tome.) 
The Price equation (see Price, 1970) is central to the book. Frank favours this formulation because it gives "an exact, complete description of evolutionary change under all conditions," that is, because it is extremely general. Price asks us to consider a population of entities that are grouped by their score on some trait $z$. Note that the entities could be genes, individuals, groups or whatever, and $z$ could of course be anything at all. But let's suppose that the entities are individual animals, and $z$ represents their degree of aggressive behaviour. Each possible value of $z$ is paired with a fitness value, $w$; for example, $z=100$ might be associated with $w=2$, which might mean, say, that highly aggressive animals tended to have two offspring on average. The population evolves in discrete generations, and the proportion of animals among the offspring generation that have a particular $z$-value is equal to the proportion of animals with the same value in the parental generation, multiplied by the relative fitness level $w / \bar{w}$ associated with it. So if 5\% of the parents have a $z$-value of 100 , the associated fitness score $w$ is 2 , and the average fitness overall is 1 , then $5 \% \times \frac{2}{1}=10 \%$ of the offspring will have $z=100$. If we look at how the average value of $z$, denoted by $\bar{z}$, will change over time, it turns out that:

$$
\bar{w} \Delta \bar{z}=\operatorname{Cov}(w, z)+\mathrm{E}(w \Delta z) .
$$

This is the Price equation, and it means that the average change per generation in mean aggression, $\Delta \bar{z}$, will be equal to the covariance between fitness and aggression, $\operatorname{Cov}(w, z)$, plus the expected change in $z$ during genetic transmission from parent to child, weighted by fitness, $\mathrm{E}(w \Delta z)$, all divided by mean parental fitness, $\bar{w}$. (I have duplicated the way Frank presents equations, with what would have been the lone term in a right-hand-side denominator-in this case, $\bar{w}$-grouped on the left hand side. This may seem strange at first, but it saves space and makes for cleaner-looking typography.) The covariance term states the relatively obvious: if there was a positive relationship between aggression and fitness in the parental generation, the mean value of aggression among the children will go up; if a negative relationship, it will go down. The second, expectation term is often ignored by other authors, because it is assumed that genetic transmission may be noisy but is, on average, reliable, and therefore the expected change in $z$ from parent to offspring will be zero. But it is clear that this term may be non-zero (e.g., if mutation is biased) and can thus affect the course of evolution.

Frank develops a great deal from this simple beginning. He begins by recursively expanding the Price equation to look at higher levels of selection: the covariance term then describes selection among groups, while the expectation term describes selection within groups and other factors. Space precludes listing all of the ways in which the ideas are developed, but some highlights for adaptive behavior researchers might well be Frank's treatment of the differing effects of large and small mutations (chapter 5), and the effects of demography and spatial dispersal as they relate to kin selection (chapter 7). Sex allocation - the question of how much to invest in sons and how much in daughters-is used throughout the book as an example problem. 


\section{Relatedness}

Kin selection and relatedness are often misunderstood, and are sometimes invoked as explanatory principles without really being measured. Frank does a lot to clarify the concept of relatedness, pointing out that it is primarily about statistical relationships between genotypes. The fact that such relationships most commonly come about because of common descent should not obscure the fact that descent, per se, is unimportant. Consider the green beard effect, in which the same gene causes a prominent phenotypic marker (i.e., a green beard) and also promotes altruistic behaviour towards others who have the marker. If this kind of phenotypic association brought about a correlation between the genotypes of social partners, then the behavioural predictions would be exactly the same as if the relationship was due to common ancestry. In chapter five, Frank develops a model of cooperation between two species just to drive home the point that it is statistical associations and not kinship itself that drives selection.

An important original contribution of the book is Frank's claim that relatedness is really two (related) things. It is both the degree to which your strategy is correlated with that of your social partner's, and the fidelity with which a characteristic is passed to offspring. We can see the difference between these two types of relatedness by looking at the success of a gene that codes for the behaviour of assisting another in raising offspring, such as helping at the nest as occurs in some bird species. Frank's point is that the cost-benefit analysis for this altruistic gene must consider first the likelihood that the recipient also carries the gene: usually this is established by kinship, for example, a sibling with $r=0.5$. Second, we have to look at the faithfulness with which this trait is copied to offspring: there is no point in helping to raise offspring that do not inherit the key characteristic from their parent(s), for example. Both of these measures have to be considered in determining whether the altruistic gene will spread (although if we assume perfect genetic transmission then we can ignore the latter). Frank suggests that the two kinds of relatedness have not previously been recognized as such because they can both be framed in the context of Hamilton's rule, that is, $r$ can be a measure of the correlation between neighbours' strategies, and it can be a measure of the probability of transmission of a rare gene, but these quantities are of course not the same thing.

After digesting this analysis, the reader may protest in hindsight that it is obvious. Frank points out that it allows us to understand a broader range of phenomena than does a narrow genealogical interpretation of kin selection. As noted above, the correlation between donor and recipient strategies may be brought about by phenotypic association and not common descent. Moreover, the trait in question may not be inherited genetically but copied with a certain fidelity by offspring (c.f. cultural evolution, Boyd $\&$ Richerson, 1985). Only from Frank's perspective could we understand the evolution of altruism in such cases.

\section{Costs and benefits of Frank's approach}

Frank makes a good case for "comparative statics." In other words, he believes we should use tools like the Price equation to identify evolutionary equilibria, then con- 
sider the effect on the equilibrium value of changes in parameter values, and finally use all this to make real-world predictions, e.g., that species A will have a higher frequency of food-sharing behaviour than species B because the degree of dispersal in A is lower. Dynamic models, although interesting, seem to go beyond our ability to test them with appropriate observations. This is, of course, a sobering reminder for our own field, where dynamic analyses of evolution are sometimes presented but rarely related back to empirical data.

Frank also sides with Grafen (1991) and Hammerstein (1996) in taking what Grafen calls the "phenotypic gambit." To accept the gambit is to ignore the (usually unknown) details of the underlying genetic system, and focus on the phenotype. In the case of social evolution, this means looking at behavioural strategies. The assumption is that even if some genetic glitch, such as heterozygote superiority, or strong epistasis, blocks progress up a fitness gradient, sooner or later a mutation will come along that changes the genetic system and allows movement to occur.

Perhaps the most obvious limitation of Frank's book is that it focuses on kin selection to the exclusion of the other hypothesized routes to cooperative social behaviour, such as mutualism, reciprocal altruism, and cultural altruism. This is not really a criticism, however, as there are strong grounds for arguing that kin selection is logically prior to these other routes (with the exception of mutualism, which is not all that interesting once identified). Reciprocal and cultural altruism are presumably relatively recent arrivals on the evolutionary scene, and require a certain level of cognitive sophistication, whereas kin selection demands no cognitive abilities whatsoever. Still, some readers may be misled by the book's title into thinking that a range of mechanisms will be discussed therein.

Frank himself identifies another limitation of the work in his concluding chapter. He points out that static methods (i.e., the identification and comparison of equilibria), having served him so well throughout the book, can unfortunately say very little about issues of conflict and power in evolution. By this Frank means problems where the evolutionary dynamics are all-important. He offers as examples host-parasite coevolution, and animal communication given a conflict of interests. In such cases, the question is whether one side or the other will gain the upper hand in the interaction, or whether the state of the system will fluctuate indefinitely. Static methods can identify the equilibrium favoured by each competitor, but cannot say which, if any, will be reached in the end.

It is interesting to note that both of the topics mentioned by Frank in this regard are the subject of numerous papers in the adaptive behavior and artificial life literature. Indeed, reading the book from the perspective of someone engaged in evolutionary simulation, I found that I was constantly comparing the two methods, considering what each one could and could not do well. For example, Frank's treatment of conditional behaviour (chapter 6), whereby an organism can change its strategy based on the perceived value of some cue, is necessarily very simplistic. This is an excellent example of a problem area where simulation techniques, with their access to flexible behavioural architectures such as neural networks, can go much further than mathematical approaches. Even though Frank does not discuss simulations, for Adaptive Behavior readers the book will implicitly highlight the tradeoff between expressiveness and tractability that exists when one is choosing between simulation and analytic 
models.

Of course, I do not expect that many in this field will experience Frank's book as some sort of road to Damascus, and promptly cast down workstations for pencils, paper and calculus texts. But if you are unsure as to whether your social simulation could be more succinctly expressed as a mathematical model, then read Foundations of Social Evolution.

\section{References}

Boyd, R., \& Richerson, P. J. (1985). Culture and the Evolutionary Process. University of Chicago Press.

Fisher, R. A. (1930). The Genetical Theory of Natural Selection. Oxford University Press.

Frank, S. A. (1998). Foundations of Social Evolution. Princeton University Press, Princeton, NJ.

Grafen, A. (1991). Modelling in behavioural ecology. In Krebs, J. R., \& Davies, N. B. (Eds.), Behavioural Ecology: An Evolutionary Approach (Third edition)., pp. 5-31. Blackwell, Oxford.

Hamilton, W. D. (1964). The genetical evolution of social behaviour. Journal of Theoretical Biology, 7, 1-52.

Hamilton, W. D. (1970). Selfish and spiteful behaviour in an evolutionary model. Nature, 228, 1218-1220.

Hammerstein, P. (1996). Darwinian adaptation, population genetics and the streetcar theory of evolution. Journal of Mathematical Biology, 34, 511-532.

Maynard Smith, J. (1989). Evolutionary Genetics. Oxford University Press.

Michod, R. E., \& Hamilton, W. D. (1980). Coefficients of relatedness in sociobiology. Nature, 288, 694-697.

Price, G. R. (1970). Selection and covariance. Nature, 227, 520-521.

Queller, D. C. (1992). A general model for kin selection. Evolution, 46, 376-380.

Williams, G. C. (1966). Adaptation and Natural Selection. Princeton University Press, Princeton, NJ. 